\title{
Generating and grading the abscopal effect: proposal for comprehensive evaluation of combination immunoradiotherapy in mouse models
}

\author{
Daniel K. Ebner ${ }^{1,2 *}$, Walter Tinganelli ${ }^{3 *}$, Alexander $\mathrm{Helm}^{3}$, Alessandra Bisio ${ }^{4}$, Palma Simoniello ${ }^{5}$, \\ Francesco Natale ${ }^{6}$, Shigeru Yamada ${ }^{1}$, Tadashi Kamada ${ }^{1}$, Takashi Shimokawa ${ }^{7}$, Marco Durante ${ }^{3}$; the \\ Abscopal Research Collaboration (ARC)
}

${ }^{1}$ Hospital of the National Institute of Radiological Sciences, National Institutes of Quantum and Radiological Science and Technology, Chiba, Japan; ${ }^{2}$ Brown University Alpert Medical School, Providence, RI, USA; ${ }^{3}$ Trento Institute for Fundamental Physics and Applications-National Institute for Nuclear Physics (TIFPA-INFN), ${ }^{4}$ Center for Integrative Biology CIBIO, University of Trento, Trento, Italy; ${ }^{5}$ University of Naples, Parthenope, Naples, Italy; ${ }^{6}$ Technical University of Darmstadt, Darmstadt, Germany; ${ }^{7}$ National Institute of Radiological Sciences, National Institutes of Quantum and Radiological Science and Technology, Chiba, Japan

Contributions: (I) Conception and design: DK Ebner, A Helm, T Shimokawa, W Tinganelli; (II) Administrative support: M Durante, S Yamada, T Kamada; (III) Provision of study materials or patients: None; (IV) Collection and assembly of data: None; (V) Data analysis and interpretation: None; (VI) Manuscript writing: All authors; (VII) Final approval of manuscript: All authors.

*These authors contributed equally to this work.

Correspondence to: Walter Tinganelli. via Aurelio Nicolodi, 52, 38133, Trento, Italy. Email: walter.tinganelli@tifpa.infn.it.

\begin{abstract}
Numerous published case reports and forthcoming clinical trials combine immunotherapy and radiotherapy with the goal of demonstrating an abscopal effect reaction. However, reports and results are varied, and a comprehensive animal study combining radiotherapy modalities and immunotherapy agents has yet to be performed. Further, clinical reports are mixed, with inconsistent generation of the effect as well as abscopal effects seen ranging from widespread disease elimination to growth stagnation of local lymph node metastasis. We propose a grading system for use in differentiating abscopal effects seen in animal and clinical trials. Further, we will conduct a comprehensive study in mice, evaluating three radiotherapeutic modalities (photon, proton, and carbon-ion) combined with five immunotherapeutic agents with differing actions. Comprehensive cell analysis will be conducted with the aim of improving upon draft models of abscopal effect generation, as well as differentiating between locoregional and systemic methods of action. Further, evaluation of radiation fractionation, as well as combination with ex vivo activated dendritic cell (DC) inoculation, will be performed. Though the abscopal effect has been reported for nearly 70 years, only recently has delineation of its mechanism seemed possible. Comprehensive evaluation of available modalities may shed light on the precise requirements for generating the effect, potentially enabling its regular usage in the treatment of disease.
\end{abstract}

Keywords: Immunotherapy; particle therapy; carbon-ion radiotherapy

Submitted Apr 06, 2017. Accepted for publication May 11, 2017.

doi: 10.21037/tcr.2017.06.01

View this article at: http://dx.doi.org/10.21037/tcr.2017.06.01 


\section{Introduction}

Current cancer treatment strategies are limited in patients presenting with late-stage, metastatic disease, with survival rates for pancreatic cancer, as an example, remaining below $10 \%$ at 5 years (1). Traditionally, once metastasis of any cancer has begun, surgical and radiotherapeutic treatment is limited to detectable disease and often considered palliative, shifting the clinical goal to prolongation of life quality over the possibility for a cure. Surgical de-bulking and radiotherapy may offer symptomatic reduction but are only able to reduce disease burden, as cancerous tissue regrows and spreads. Generalized chemotherapies are employed in an attempt to slow down disease progression, with total disease remission and cure viewed as unusual if not impossible. Further, aggressive irradiation or chemotherapy treatment of metastatic disease can result in undetected secondary tumors arising following treatment of the primary, an as-yet mechanistically unexplained phenomenon known as the Phoenix Rising effect (2).

The abscopal effect is the full systemic challenge of malignant disease by the host system, resulting in disease stagnation, retardation, and/or elimination. Existing case reports indicate that the immune system in at least some subset of patients can fully eliminate widespread cancerous disease if properly stimulated (3). To date, a detailed mechanism has yet to be conclusively developed for induction of the abscopal effect. Expanded understanding of this method would provide a powerful tool in the fight against cancer, particularly with the potential of personalized immunotherapy on the horizon. As such, discovery of the mechanism by which the body is able to uncover the abscopal tumor in particular is paramount.

Here, we briefly review the literature with regard to the abscopal effect, propose a grading scale for evaluation of reported effects, as well as outline an upcoming study on the combination of immunotherapy and radiotherapy, with aim to elucidate the molecular mechanisms of the abscopal effect.

\section{Combination immunoradiotherapy}

Tumor cells often produce and expose antigens that can potentially trigger an immune response and consequent tumor elimination. The antigens are recognized and endocytosed by antigen-presenting cells (APCs), which in turn process and present the antigens to $\mathrm{T}$ cells, triggering an immune response culminating in the targeting of the tumor (4). As tumors are subsequently destroyed, a selective pressure is created wherein tumors presenting detectable antigens are eliminated, leaving only those tumors that do not readily identify themselves to the immune system. This process, termed immune-editing (4), enables the tumor to adapt to and eventually escape immune system attack, expanding and metastasizing.

A number of key players have been identified in antitumor immune system activation, which our group reviewed previously (4). Calreticulin (CRT) is a chaperone molecule usually present in the endoplasmatic reticulum that is responsible for preventing misfolded protein transport to the Golgi apparatus. Upon stress stimuli, including radiation exposure, CRT translocates to the cell surface and is presented to dendritic cells (DC) (4). High mobility group box 1 (HMGB1), meanwhile, is a nuclear molecule that usually interacts with histones, transcription factors, and nucleosomes, and is responsible for DNA organization and transcription. It further plays a major role in inflammation and is released by necrotic cells, activated macrophages, and DC, functioning as a pro-inflammatory cytokine following cancer treatment (4).

Balance between activation and suppression of the immune system is required for a proper immune response. Increased levels of prostaglandin E2 (PGE2) induces the conversion of $\mathrm{CD}^{+} \mathrm{T}$ cells into regulatory $\mathrm{T}$ (Treg) cells, a subpopulation of $\mathrm{CD}^{+}$cells known to suppress immune responses and maintain immune homeostasis in physiological conditions. However, Tregs in the tumor microenvironment contribute to the tumor ability to evade immunosurveillance, and their presence at the tumor site correlates with poorer patient outcomes. Tregs are involved in the development of different malignancies such as prostate (5), multiple myeloma (6), breast cancer (7), glioma (8) and malignant melanoma (9). As a therapeutic target, Tregs have been found to be more radioresistant than other lymphocyte subpopulations such as $\mathrm{T}$ or B cells (10-11). This may be mediated by down-regulation of proapoptotic proteins and up-regulation of anti-apoptotic proteins such as BCL-2, as demonstrated by Qu and colleagues (12). Moreover, radiotherapy has been found to increase the recruitment of pre-existing Tregs to local tumor microenvironment, causing local immunosuppression in the tumor site (13). There is further evidence that even minor differences in radiation dose may determine the balance between a tolerogenic and efficient antitumor immune response, changing Treg/effector $\mathrm{T}$ cell ratios (14).

Immunotherapy serves to target players in the immune response pathway, such as cytotoxic T-lymphocyte- 
associated protein 4 (CTLA-4) (15), programmed cell death protein 1 (PD-1), and programmed death-ligand 1 (PD-L1) (16), with the goal of upregulating tumor targeting and disrupting the immunosuppressive tumor microenvironment. Also known as cluster of differentiation 152 (CD152), CTLA-4 is a protein receptor and member of the immunoglobulin superfamily, and serves as an immune system checkpoint. It is constitutively expressed by Treg cells and upregulated in conventional $\mathrm{T}$ cells after activation, working as an off switch when linked to CD80 or CD86 antigen presenting cells, which serve as gateways to immune system activation. As such, CTLA-4 primarily serves to downregulate the immune response, and blockage of this molecule is thought to be a possible target for immune system activation and reduction of the suppressive tumor microenvironment. Though many are under development, ipilimumab is the only FDA-approved CTLA4 inhibitor available today, which is used principally against melanoma (17). Meanwhile, PD-L1, or cluster of differentiation 274 (CD274), is a $40 \mathrm{kDa}$ transmembraneous protein that suppresses the immune system during important systemic events, like pregnancy. In general, the immune system reacts to non-self-antigens by proliferating $\mathrm{CD}^{+} \mathrm{T}$ cells, which then attack the target. The binding of PD-L1 with the T-cell receptor PD-1 transmits an inhibitor signal that is then able to reduce proliferation and the immune response. The downstream effects of this pathway mitigate the accumulation of foreign antigenspecific $\mathrm{T}$ cells in the lymph nodes by causing apoptosis, further regulated by the gene Bcl-2 (18). PD-L1 has been found to be upregulated in many cancers (19), which likely contributes to tumor evasion of the immune system (20).

Radiotherapy, on the other hand, is generally thought of as an immune-activating agent (21) with some downstream immunosuppressive effects as mentioned above. Irradiation disrupts the tumor microenvironment and, in theory, (re)enables immune response in the region. Though radiotherapy has both immune-activating and immunerepressing properties, current research suggests that irradiation can convert the tumor into an in situ vaccine, serving as a hub for immune system activation (22). This activation can, in theory, be expanded and further amplified with proper immunotherapeutic application. The activated immune cells travel through the lymphatic system, flowing downstream to the heart where, after passing through the pulmonary circulation, they are dispersed throughout the body. This process is thought to underlie the development of the systemic response seen in the abscopal effect; however, mechanistic understanding of how this operates is limited. Further, distant tumors presumably remain in immunosuppressive environments; how these environments are disturbed and disease uncovered remains unsolved.

\section{Photon and particle radiotherapy}

Multiple forms of radiation therapy are in use today, though they predominantly fall into two major categories: photon ("conventional") and particle irradiation. Photon irradiation is delivered using $\mathrm{X}$-rays and involves the transference of energy via electrons to both directly disrupt target cell DNA, as well as to generate free radicals through electron fixing, causing a chemical effect on the target. This irradiation tends to deliver a gradually diminishing effect from the skin surface to skin exit, and so today dose is generally collated from multiple angles or continuously in an arc with beams of varying intensities, delivering target dose while minimizing healthy tissue irradiation. Particle radiotherapy, meanwhile, consists of proton or ion beams. Unique to particles is an interaction with environmental matter inversely proportional to speed/energy: as the particle slows, it delivers more ionization energy to surrounding tissue, eventually reaching an asymptotic point at which particle motion ceases, and all energy is released. This point is called the Bragg peak, and particles are thereby characterized by low entry and essentially nonexistent exit dose. Treatment delivery involves substantial cost, and so centers are uncommon in comparison with photon. Particle therapy may be roughly divided into proton centers, increasingly available at academic centers worldwide, and heavy-ion therapy, which is limited to a handful of centers. Heavy-ion therapy today consists mainly of carbon-ion, with possible clinical implementation of oxygen-ions in the near future (23). Beyond dose distributive benefits, heavy ions further offer differential deposition of energy in relation to the target, termed their linear energy transfer. Carbon ions, possessing a LET of 3 compared to 1.1 for proton and 1.0 (standardized) for photon, thereby has additional biological effects in target tissue, including a substantially enhanced ratio of double strand breaks. Preliminary evidence suggests that the carbon-ion beam may offer enhanced potential for generation of an abscopal effect via induction of immunogenic cell death (4).

\section{Grading the abscopal effect}

When considering the downstream flow of the abscopal 
Table 1 Prototype experimental abscopal effect grading system

\begin{tabular}{ll} 
Primary tumor inflammation and shrinkage & Grade 0 \\
Reduction of spreading metastases & Grade 1A \\
New cell tumor rejection after injection & Grade 1B \\
Secondary tumor inflammation & Grade 1C \\
Secondary tumor shrinkage & Grade 2A \\
Total systemic elimination of all disease & Grade 2B \\
No recurrences & Grade 3 \\
\hline
\end{tabular}

Table 2A Prototype clinical abscopal effect grading system

\begin{tabular}{ll} 
Primary tumor inflammation and shrinkage & Grade OA \\
$\begin{array}{l}\text { Anti-metastatic effect. (An apparent clinical } \\
\text { decrease in number of metastases at typical } \\
\text { downstream sites, such as the lungs or liver) }\end{array}$ & Grade 0B \\
Locoregional lymph disease stabilization & Grade 1A \\
Regression & Grade 1B \\
Elimination & Grade 1C \\
Distant lymph node disease stabilization & Grade 2A \\
Regression & Grade 2B \\
Elimination & Grade 2C \\
Non-lymph downstream metastasis stabilization & Grade 3A \\
Regression & Grade 3B \\
Elimination & Grade 3C \\
Systemic metastasis stabilization (req. traversal of & Grade 4A \\
systemic circulation) & \\
Regression & Grade 4B \\
Elimination & Grade 4C \\
Total elimination of disease & Grade 5 \\
\hline
\end{tabular}

effect, it is intuitive that the process involved in tumor regression in a regional lymph node may differ from that of systemic remission of disease outside the lymphatic system, or between pulmonary and systemic circulation. To investigate such processes, for example, an amenable animal model will undergo intracutaneous inoculation of tumor cells to both hind limbs. Thereafter, irradiation of a single limb will be conducted, with subsequent evaluation of the tumor on the non-irradiated limb following any adjuvant pharmaceutical inoculation. Using this example, however, an immune response-locally activated in the
Table 2B Clinical grading usage examples

Total widespread disease elimination in a melanoma Grade 5 patient

Mediastinal lymph node metastasis from colorectal Grade 2B cancer that shrinks following treatment

Regression of systemic metastasis in a head-and- Grade 4B neck case

Stagnation of liver metastasis from a pancreatic Grade 3A cancer primary

Elimination of single distant lymph node metastasis, Grade 5/2C leaving patient disease free

irradiated hind limb-would have to enter the arterial or venous blood circulation, or lymphatics, in order to pass into the system. From these entrance points, the response factors would travel to the heart (via the liver, depending on tumor location), then through the pulmonary circulation before at last passing back into the systemic circulation. Along the way, an unknown number of factors may play a role in amplifying or diminishing the response to the irradiated tumor; conversion of the tumor into an in-situ vaccine and non-total elimination of the local tumor may thus be necessary to build and sustain an immune response capable of systemic proliferation. Finally, an experimental model of the abscopal effect differs from a clinical model in terms of follow-up, and so the abscopal effects seen in each may be better graded separately.

With this in mind, we propose the following prototype abscopal effect grading systems, representing the differential nature of abscopal effect responses that may be noted. An experimental scale for evaluation of animal models is provided in Table 1, while a potential clinical model is provided in Table $2 A, B$.

The experimental model focuses primarily on readily definable parameters traceable in a small animal model, such as a mouse, where technological limits preclude the regular follow-up that may be seen in clinical models. The progression of grades is based on preliminary data: in this case, a "true" experimental abscopal effect may be considered grade $2+$.

Usage examples of the clinical scale are provided in Table 2B. Of particular note, grades are subcategorized based on the degree of abscopal effect noted in total response, as seen in the last row of Table $2 B$, so as to preserve the degree of reaction for research and review purposes. Cases, 


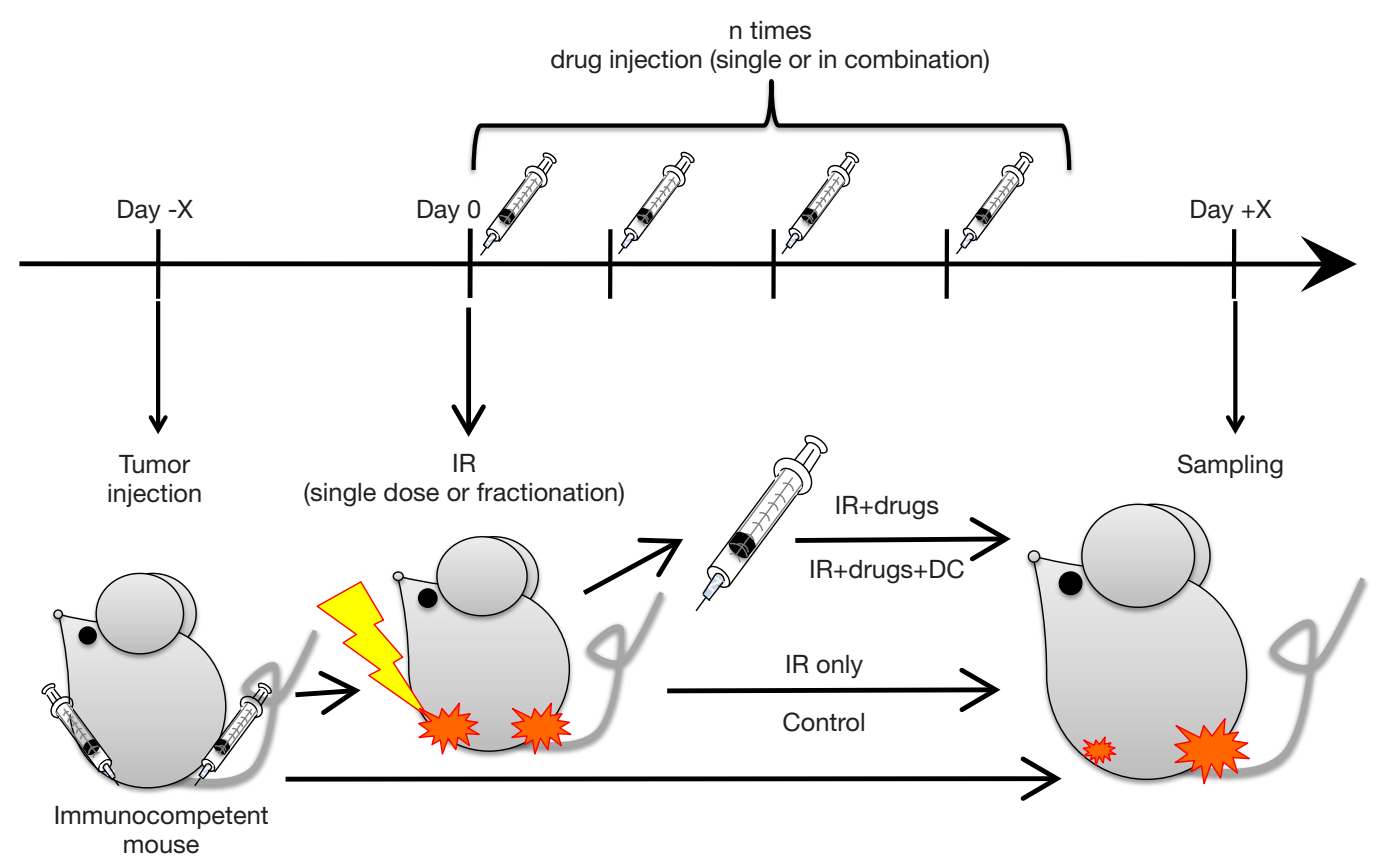

Figure 1 The general schematic for the single and multi-drug inoculation trials. Prior to treatment, tumor cells will be inoculated in both hind limbs, with one limb lagging a day behind the other (constituting the smaller "abscopal tumor"). The primary tumor will be irradiated, with single or, later, combination immunotherapeutics delivered thereafter.

in which disease is contiguous with the primary (locallyadvanced disease) but regresses outside the radiation field, or non-contiguous but marginally-irradiated, should be noted as such when grading is reported. Upon disease recurrence, grade 5 disease may be restaged.

This preliminary scale can serve as a guide for evaluation of both experimental and clinical abscopal effects for comparative purposes, and may evolve with improved mechanistic and clinical understanding of the presentation of the effect in laboratory and clinical settings.

\section{Protocol}

If understanding of combination immunoradiotherapy is extended to include regular generation of the abscopal effect, medicine would experience a significant leap forward in cancer care. The existence of multiple radiation modalities, as well as ongoing release of immunotherapy agents, calls for a comprehensive combination study, which the ARC Group is currently performing. This protocol uses the ARC Experimental Abscopal Effect grading model (Table 1). Here, we include an overview of our ongoing evaluation protocol.

\section{Single immunotherapy agent protocol}

Initial work consists of testing combinations of a single immunotherapeutic with photon, proton, and carbon-ion irradiation, so as to delineate which agents appear to (best) generate an immune response. Murine tumor cells will be injected subcutaneously into an immune-competent mouse left hind limb before irradiation, with time allowed for tumor growth dependent on the cell model doubling time. Shortly following left limb inoculation, an identical number of cells will be injected into the right hind limb and will go unirradiated, constituting the "abscopal tumor."

After tumor growth, the left-limb tumor will be irradiated with 10 Gy carbon-ion, 30 Gy X-ray, or 27 Gy of protons, scaled to produce approximately equivalent relative biological doses (RBE). Immediately following irradiation, a single immunotherapeutic agent will be injected intraperitoneally (Figure 1). In initial trials, we intend to test a selective nonsteroidal anti-inflammatory, an Nrf2 pathway activator and NF-kB inhibitor, a CTLA-4 inhibitor, an IgG4 anti-PD-1 monoclonal antibody, and an adenosine A2A receptor antagonist, constituting five separate agents. This results in 24 groups, including agent combinations 
with radiotherapy modalities as well as control groups.

The ability of individual agents in combination with radiotherapy to generate a grade $1 \mathrm{C}$ systemic abscopal effect will constitute the primary endpoint, as measured by antitumor effect seen in the right hind limb abscopal tumor.

\section{Combination immunotherapeutic evaluation}

This initial evaluation will be followed by an identically structured experiment, in which agent cocktail combinations, specifically those found effective in the initial evaluation, are tested. Biopsies and blood will be extracted and analyzed in attempt to discover any biomolecular changes occurring in animals demonstrating abscopal effects versus those animals that do not express the effect.

\section{DC inoculation evaluation}

In addition to the previous cocktail agent combination therapy, radiation combinations will similarly be used to evaluate activation of DC populations. In this case, a longer pre-irradiation tumor growing time will be allowed, with immature DC isolated from a tail vein sample taken several days following tumor inoculation. Thereafter, a tumor biopsy sample will be taken, with tumor cells separated by cytofluorimetry or DEPArray. These cells will be irradiated. The isolated immature DC will then be activated ex vivo by culturing with DC-maturation adjuvant and tumorirradiated cells (24), before being reintroduced to the host animal. Future experiments may evaluate the combination of ex vivo DC inoculation with immuno-radiotherapeutic treatment.

\section{Evaluation of fractionation}

Recent evidence has raised the question of radiotherapeutic fractionation and its impact on immunogenic response, specifically whether hypofractionation may reduce the likelihood of abscopal effect generation (25). Alternatively, extended fractionation may have an effect secondary to irradiation of the invading immune system response. Protocols from the single-agent and multi-agent trials described above that are successful in inducing an abscopal effect will be further evaluated by varying the number of fractions in which radiation is delivered. Future work will additionally take agent inoculation timing (prior to or following irradiation) into account.

\section{Discussion}

The abscopal effect is a rare phenomenon that if understood and reproduced would radically change cancer therapy, opening the door to late-stage treatment and potential cure of disease. In the existing case literature, even in those patients with particularly poor prognosis, the effect resulted in months to years of added life, and at times even allowed for total disease remission, following a single palliative radiotherapy treatment with and without immunotherapy (26-27). While this suggests that the abscopal effect is a natural capacity that radiation and immunotherapy can activate and amplify, and while clinical trials on combinations are increasing at a frantic pace, the basics of an activation mechanism remain elusive. This may owe to dependency of the effect on specific as-yet-unknown hosttumor (epi)genetic factors that are not currently evaluated clinically, particularly due to the widespread heterogeneity of tumor tissues; that is, a general mechanism for the abscopal effect may not exist, and patient-specific precision treatment may be necessary. To this end, comprehensive animal studies in combination with clinical results must be considered.

The term abscopal effect is descriptive and reactive in nature, and may be an umbrella term describing a range of disparate mechanisms operating simultaneously, dependent on histology, location, abscopal-tumor-distance, among others. To begin to tease out these differences, our group here proposes a prototype grading scale for animal and clinical abscopal reactions, delineated from the perceived flow of immunogenic factors from the treated primary through blood and lymph circulations. This is particularly valuable as case reports note regional lymph-node regression, while animal studies use distant systemic disease that requires full traversal of the circulatory system; the two cases may function under differing mechanisms, and comparative evaluation will be needed.

As discussion of personalized immunotherapy increases, we can envision patients treated with comparatively limited radiation in combination with designed tumor-host-specific immunotherapeutics, priming their immune system to eradicate all disease. It is through evolving mechanistic understanding of how the immune system operates in these exceptional cases that we may at last be able to open the door to the next stage of disease treatment. To this end, comprehensive evaluation of available modalities may shed light on the precise requirements for generating the 
abscopal effect and widespread immune system activation, potentially enabling its regular usage in the treatment of disease.

\section{Acknowledgements}

None.

\section{Footnote}

Conflicts of Interest: The authors have no conflicts of interest to declare.

\section{References}

1. Nitecki SS, Sarr MG, Colby TV, et al. Long-term survival after resection for ductal adenocarcinoma of the pancreas. Is it really improving? Ann Surg 1995;221:59-66.

2. Allen CP, Tinganelli W, Sharma N, et al. DNA Damage Response Proteins and Oxygen Modulate Prostaglandin E2 Growth Factor Release in Response to Low and High LET Ionizing Radiation. Front Oncol 2015;5:260.

3. Huang Q, Li F, Liu X, et al. Caspase 3-mediated stimulation of tumor cell repopulation during cancer radiotherapy. Nat Med 2011;17:860-6.

4. Ebner DK, Tinganelli W, Helm A, et al. The Immunoregulatory Potential of Particle Radiation in Cancer Therapy. Front Immunol 2017;8:99.

5. Yan L, Da Silva DM, Verma B, et al. Forced LIGHT expression in prostate tumors overcomes Treg mediated immunosuppression and synergizes with a prostate tumor therapeutic vaccine by recruiting effector $\mathrm{T}$ lymphocytes. Prostate 2015;75:280-91.

6. Favaloro J, Brown R, Aklilu E, et al. Myeloma skews regulatory $\mathrm{T}$ and pro-inflammatory $\mathrm{T}$ helper 17 cell balance in favor of a suppressive state. Leuk Lymphoma 2014;55:1090-8.

7. Watanabe MA, Oda JM, Amarante MK, et al. Regulatory $\mathrm{T}$ cells and breast cancer: implications for immunopathogenesis. Cancer Metastasis Rev 2010;29:569-79.

8. Grauer OM, Nierkens S, Bennink E, et al. CD4+FoxP3+ regulatory $\mathrm{T}$ cells gradually accumulate in gliomas during tumor growth and efficiently suppress antiglioma immune responses in vivo. Int J Cancer 2007;121:95-105.

9. Nishikawa H, Sagakuchi S. Regulatory T cells in cancer immunotherapy. Curr Opin Immunol 2014;27:1-7.

10. Bogdándi EN, Balogh A, Felgyinszki N, et al. Effects of low-dose radiation on the immune system of mice after total-body irradiation. Radiat Res 2010;174:480-9.

11. Komatsu N, Hori S. Full restoration of peripheral Foxp3 regulatory $\mathrm{T}$ cell pool by radioresistant host cells in scurfy bone marrow chimeras. Proc Natl Acad Sci USA 2007;104:8959-64.

12. Qu Y, Jin S, Zhang A, et al. Gamma-ray resistance of regulatory CD4+CD25+Foxp3+ T cells in mice. Radiat. Res 2010;173:148-57.

13. Malchow S, Leventhal DS, Nishi S, et al. Aire-dependent thymic development of tumor-associated regulatory $\mathrm{T}$ cells. Science 2013;339:1219-24.

14. Battaglia A, Buzzonetti A, Martinelli E, et al. Selective changes in the immune profile of tumor-draining lymph nodes after different neoadjuvant chemoradiation regimens for locally advanced cervical cancer. Int J Radiat Oncol Biol Phys 2010;76:1546-53.

15. Mocellin S, Nitti D. CTLA-4 blockade and the renaissance of cancer immunotherapy. Biochim Biophys Acta 2013;1836:187-96.

16. Zitvogel L, Kroemer G. Targeting PD-1/PD-L1 interactions for cancer immunotherapy. Oncoimmunology 2012;1:1223-5.

17. Pardoll DM. The blockade of immune checkpoints in cancer immunotherapy. Nat Rev Cancer 2012;12:252-64.

18. Chemnitz JM, Parry RV, Nichols KE, et al. SHP-1 and SHP-2 associate with immunoreceptor tyrosine-based switch motif of programmed death 1 upon primary human $\mathrm{T}$ cell stimulation, but only receptor ligation prevents $\mathrm{T}$ cell activation. J Immunol 2004;173:945-54.

19. Thompson RH, Gillett MD, Cheville JC, et al. Costimulatory B7-H1 in renal cell carcinoma patients: Indicator of tumor aggressiveness and potential therapeutic target. Proc Natl Acad Sci U S A 2004;101:17174-9.

20. Silginer M, Nagy S, Happold C, et al. Autocrine activation of the IFN signaling pathway may promote immune escape in glioblastoma. Neuro Oncol 2017. [Epub ahead of print].

21. Formenti SC, Demaria S. Systemic effects of local radiotherapy. Lancet Oncol 2009;10:718-26.

22. Reynders K, Illidge T, Siva S, et al. The abscopal effect of local radiotherapy: using immunotherapy to make a rare event clinically relevant. Cancer Treat Rev 2015;41:503-10.

23. Durante M, Tommasino F, Yamada S. Modeling Combined Chemotherapy and particle Therapy for Locally Advanced Pancreatic Cancer. Front Oncol 2015;5:145.

24. Palucka K, Banchereau J. Cancer immunotherapy via dendritic cells. Nat Rev Cancer 2012;12:265-77. 
25. Dewan MZ, Galloway AE, Kawashima N, et al. Fractionated but not single-dose radiotherapy induces an immune-mediated abscopal effect when combined with anti-CTLA-4 antibody. Clin Cancer Res 2009;15:5379-88.

26. Postow MA, Callahan MK, Barker CA, et al. Immunologic correlates of the abscopal effect in a patient with melanoma. N Engl J Med 2012;366:925-31.

27. Abuodeh Y, Venkat P, Kim S. Systematic review of case reports on the abscopal effect. Curr Probl Cancer 2016;40:25-37.
Cite this article as: Ebner DK, Tinganelli W, Helm A, Bisio A, Simoniello P, Natale F, Yamada S, Kamada T, Shimokawa T, Durante M; the Abscopal Research Collaboration (ARC). Generating and grading the abscopal effect: proposal for comprehensive evaluation of combination immunoradiotherapy in mouse models. Transl Cancer Res 2017;6(Suppl 5):S892S899. doi: 10.21037/tcr.2017.06.01 